\title{
Influência da biomassa de leguminosas sobre a produção de repolho em dois cultivos consecutivos
}

\author{
Thiago de O Vargas ${ }^{1}$; Ellen Rúbia Diniz ${ }^{1}$; Ricardo Henrique S Santos ${ }^{1}$; Carlos Túlio de A Lima ${ }^{1}$; Segundo \\ Urquiaga $^{2}$; Paulo Roberto Cecon ${ }^{3}$ \\ 'UFV-Dep ${ }^{\text {to }}$ Fitotecnia, Av. P.H. Rolfs s/nº , 36570-000 Viçosa-MG; vargasthiago@yahoo.com.br; ellenrubiadiniz@hotmail.com;

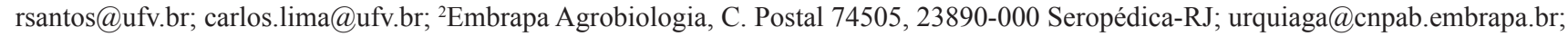 \\ ${ }^{3}$ UFV-Dep ${ }^{\text {to }}$ Informática; cecon@ufv.br
}

\section{RESUMO}

Em estudos de avaliação do desempenho de culturas em sequência à adubação verde, geralmente não se considera o efeito das raízes dos adubos verdes. Entretanto, tais efeitos podem contribuir para maior entendimento científico da adubação verde. Foram avaliados o efeito da raiz e da parte aérea das leguminosas Crotalaria juncea e Canavalia ensiformis sobre a produção do repolho em dois cultivos. $\mathrm{O}$ experimento foi instalado em esquema fatorial $(2 \times 3)+2$, sendo 2 espécies de leguminosas e 3 partes das plantas (raiz, parte aérea ou planta inteira) e 2 tratamentos adicionais (adubação mineral com $100 \%$ ou $50 \%$ da dose recomendada de $\mathrm{N}$ ), em blocos casualizados com 4 repetições. A parte aérea do adubo verde foi aplicada em superfície. O cultivo sobre a massa da parte aérea ou da planta inteira de crotalária ou feijão de porco reduziu a necessidade de adubação com N-mineral em 50\%, mas a produção de repolho foi reduzida com o cultivo sobre o sistema radicular de feijão de porco. Áreas em que foram deixadas a parte aérea ou a planta inteira de crotalária ou feijão de porco resultaram em maiores efeitos residuais sobre o segundo cultivo que a adubação com $\mathrm{N}$-mineral. O cultivo sobre as raízes destas leguminosas não resultou em efeito residual diferente da adubação mineral com 50 ou $100 \%$ da recomendação da adubação N.

Palavras-chave: Brassica oleracea var. capitata, Crotalaria juncea, Canavalia ensiformis, adubação verde, efeito residual, nitrogênio, raiz, parte aérea.

\section{ABSTRACT \\ Influence of the biomass of legumes on cabbage yield held in two consecutive crops}

In studies assessing the performance of crops in response to green manure generally are not considered the effect of roots. However, such effects may contribute to increase the scientific understanding towards this technique. The effect of the root and of the aboveground part of two legume species, Crotalaria juncea and Canavalia ensiformis, were evaluated on cabbage yield in two crops. The experiment was arranged in randomized blocks with 4 replications in a factorial design $(2 \times 3)+2$ (2 legumes species - Crotalaria juncea (sunnhemp) and Canavalia ensiformis (jack beans), 3 plant parts (root, aboveground part and the whole plant) and 2 additional treatments (mineral fertilizer $100 \%$ and $50 \%$ of recommended $N$ ). The green manure was applied to the surface. The cultivation with the aboveground part or the whole plant of sunnhemp or jack beans reduced the necessity of fertilization with mineral $\mathrm{N}$ in $50 \%$, but the cabbage yield was reduced with the cultivation with the root system of jack beans. Areas with the aboveground part or the whole plant of sunnhemp or jack beans presented higher residual effects on the second crop with mineral $\mathrm{N}$ fertilizer. The cultivation with the roots of these legumes resulted in no residual effect different from mineral fertilizer with 50 or $100 \%$ of recommended $\mathrm{N}$.

Keywords: Brassica oleracea var. capitata, Crotalaria juncea, Canavalia ensiformis, green manure, residual effect, nitrogen, root, shoot.

\section{(Recebido para publicação em 29 de outubro de 2010; aceito em 29 de novembro de 2011) (Received on October 29, 2010; accepted on November 29, 2011)}

$E^{\prime \prime}$ m Minas Gerais a produção anual de repolho foi de $52.443 \mathrm{t}$ em 2006 (IBGE, 2011) com produtividade média de $40 \mathrm{t} \mathrm{ha}^{-1}$ (Vidigal et al., 2007). Sob manejo orgânico, onde são utilizados adubos orgânicos diversos e adubos verdes, Souza \& Resende (2006) citam produtividades médias de 55,32 $\mathrm{t} \mathrm{ha}^{-1}$. Entretanto, poucos estudos foram feitos para essa cultura com o uso de adubos verdes.

O uso de adubos verdes como adubação complementar às culturas é uma alternativa para reduzir as limitações do uso de fertilizantes minerais ou de estercos e compostos orgânicos (Diniz et al., 2007) com resultados positivos. Diniz et al. (2007), estudando brócolis, verificaram que a aplicação de 8,64 tha-1 de matéria seca do adubo verde com 12 t ha ${ }^{-1}$ de matéria seca de composto orgânico aumentou o teor do $\mathrm{N}$ mineral no solo tanto quanto o fertilizante mineral (160 kg ha-1 de $\mathrm{N}$, na forma de sulfato de amônio) ou $25 \mathrm{t} \mathrm{ha}^{-1}$ de matéria seca de composto orgânico. Para o repolho, os adubos verdes Crotalaria juncea, Mucuna aterrima ou Canavalia ensiformis acrescidos de composto orgânico, resultaram na obtenção de cabeças de repolho com peso comercial de $1,4,1,2$ e $1,2 \mathrm{~kg}$, respectivamente, demonstrando potencial da adubação verde na sua produção (Fontanétti et al., 2006). Esses resultados foram superiores aos $0,9 \mathrm{~kg}$ encontradas por Oliveira et al. (2001), trabalhando com adubação orgânica para a produção de repolho com a dose de $41,0 \mathrm{t} \mathrm{ha}^{-1}$ de esterco bovino e foram semelhantes aos obtidos por Oliveira et al. (2005), que encontram $1,3 \mathrm{~kg}$ para repolho cultivado em áreas com pré-cultivo de crotalária.

Na maioria dos estudos de avaliação do desempenho de culturas em sequência à adubação verde, geralmente, não se considera o efeito das raízes, sendo que essas frações ou são ignoradas ou 
subestimadas (Khan et al., 2002). Estudos demonstraram que o $\mathrm{N}$ derivado da mineralização de raízes contribuiu para o aumento de 35 e $44 \%$ no teor de $\mathrm{N}$ -residual no solo e constituía de 79-85\% de todo o N subterrâneo (Mayer et al., 2003). Já em outros estudos (Sawatsky \& Soper, 1991; Jensen, 1996) constatou-se que do total de nitrogênio do solo, $47 \%$ eram originados das raízes.

Considera-se portanto, que o conhecimento dos efeitos das diferentes partes das leguminosas sobre a produção das culturas pode contribuir para o maior entendimento científico da adubação verde. Os objetivos deste trabalho foram avaliar o efeito da raiz, da parte aérea ou da planta inteira de Crotalaria juncea e Canavalia ensiformis, bem como seus efeitos residuais sobre a produção do repolho em condições de campo.

\section{MATERIAL E MÉTODOS}

$\mathrm{O}$ experimento foi realizado em Viçosa-MG, de dezembro de 2007 a dezembro de 2008 em um Cambissolo (Embrapa, 1999) com as seguintes características químicas na camada de 0-20 cm: $\mathrm{pH}$ em água $(1: 2,5)=5,49 ; \mathrm{P}=$ $30 \mathrm{mg} \mathrm{dm}^{-3} ; \mathrm{K}=176 \mathrm{mg} \mathrm{dm}^{-3} ; \mathrm{Ca}=3,04$ $\mathrm{cmol}_{\mathrm{c}} \mathrm{dm}^{-3} ; \mathrm{Mg}=0,50 \mathrm{cmol}_{\mathrm{c}} \mathrm{dm}^{-3} ; \mathrm{Al}=$ $0,0 \mathrm{cmol}_{\mathrm{c}} \mathrm{dm}^{-3} ; \mathrm{MO}=2,66 \mathrm{dag} \mathrm{kg}^{-1}$.

$\mathrm{O}$ experimento foi disposto em esquema fatorial $(2 \times 3)+2$, sendo 2 espécies de leguminosas empregadas como adubação verde: $C$. juncea (CR) ou $C$. ensiformis (FP) e 3 partes das plantas: raiz (RA), parte aérea (PA) ou planta inteira (PI) e 2 tratamentos adicionais: adubação mineral com 100\% (AM100) ou 50\% (AM50) da dose recomendada de $\mathrm{N}$ para a cultura do repolho, em blocos casualizados com 4 repetições, totalizando 32 parcelas. Os tratamentos foram: AM100, AM50, CRRA+AM50 (Crotalária (RA) + 50\% AM N), CRPA+AM50 (Crotalária (PA) $+50 \%$ AM N), CRPI+AM50 (Crotalária $(\mathrm{PI})+50 \%$ AM N), FPRA+AM50 (Feijão de porco (RA) + 50\% AM N), FPPA+AM50 (Feijão de porco (PA) $+50 \%$ AM N) e FPPI+AM50 (Feijão de porco $(\mathrm{PI})+50 \% \mathrm{AM} \mathrm{N})$. A massa das raízes foi estimada com base na proporção raiz:parte aérea obtida em experimento conduzido em vasos (Var- gas, 2009), tendo sido determinadas as razões de 0,92 e 0,25 para crotalária e feijão de porco, respectivamente. Antecedendo o plantio das leguminosas, foi realizada uma adubação com superfosfato simples nos sulcos de plantio na dose de $400 \mathrm{~kg} \mathrm{ha}^{-1}$ de $\mathrm{P}_{2} \mathrm{O}_{5}$. As sementes dos adubos verdes foram inoculadas com bactérias apropriadas do gênero Rhizobium, sendo utilizadas 25 sementes por metro linear para crotalária e 7 sementes por metro linear para feijão de porco e o espaçamento entre linhas foi de $80 \mathrm{~cm}$.

As plantas de adubos verdes foram cortadas ao nível do solo aos 118 dias após o plantio. Uma amostra da massa da parte aérea foi pesada e foram retiradas subamostras, as quais foram pesadas, lavadas e submetidas à secagem em estufa de ventilação forçada de ar a $65^{\circ} \mathrm{C}$, até atingirem massa constante, sendo pesadas para a quantificação da matéria seca. Os macronutrientes foram extraídos por digestão nitroperclórica ou sulfúrica (Tedesco et al., 1995). Foi determinada a fixação biológica de $\mathrm{N}$ atmosférico (FBN) da crotalária e do feijão de porco pela técnica de abundância natural de ${ }^{15} \mathrm{~N}$ (Boddey et al., 1994) com auxílio de um espectrômetro de matéria Finnigan MAT $^{\circledR}$, modelo Delta Plus ${ }^{\circledR}$, o qual estimou o $\delta^{15} \mathrm{~N}$ das amostras.

Para a produção do repolho no primeiro cultivo em abril de 2008 , foi feito o transplantio de mudas do híbrido 'Matsukaze' nas mesmas linhas de plantio das leguminosas, um dia após o seu corte. As plantas estavam espaçadas de $80 \times 50 \mathrm{~cm}$, totalizando 25 plantas por parcela experimental. A parcela útil foi formada pelas 9 plantas centrais. $\mathrm{O}$ repolho foi adubado com 150 ou $75 \mathrm{~kg}$ de $\mathrm{N} \mathrm{ha}^{-1}$, correspondentes a $100 \%$ ou $50 \%$ da dose recomendada, aplicados no plantio visando reduzir a interferência deste nutriente no processo de decomposição da massa das leguminosas e mineralização dos nutrientes presentes. O fertilizante nitrogenado foi adicionado imediatamente ao lado da muda, na forma de uréia, em um pequeno orifício a uma profundidade de $1 \mathrm{~cm}$, conforme descrito por Pereira (2007). O P foi todo aplicado no plantio das leguminosas na forma de superfosfato simples. A dose de $\mathrm{K}$ foi de $100 \mathrm{~kg} \mathrm{ha}^{-1}$ de $\mathrm{K}_{2} \mathrm{O}$ na forma de cloreto de potássio, parcelada em três vezes, no plantio, e aos 37 e 57 dias após. O combinado de micronutrientes FTE BR-12 foi aplicado ainda na dose de $62,5 \mathrm{~kg} \mathrm{ha}^{-1}$ na cova de plantio do repolho. As plantas de repolho foram cultivadas por 92 dias.

O segundo cultivo foi iniciado em agosto com o plantio do mesmo híbrido de repolho após a colheita do primeiro cultivo, na mesma área, sem qualquer adubação. A vegetação presente na área foi capinada e todo o material foi deixado na própria área de cultivo. As plantas de repolho foram cultivadas no campo por 118 dias. Apenas nos dois cultivos de repolho foi utilizada irrigação por aspersão quando necessária.

Nos dois ciclos, foram avaliadas a produção de matéria fresca e seca da cabeça e total das plantas de repolho (cabeça, folhas e caule) e o diâmetro da cabeça. As médias dos tratamentos foram comparadas com a testemunha utilizando-se o teste de Dunnett a 5\% de probabilidade, adotando-se como testemunha os tratamentos AM100 ou AM50. A comparação entre as partes das plantas e as espécies de leguminosas foi realizada pelo teste de Tukey a 5\% de probabilidade. As análises foram realizadas no Sistema para Análises Estatísticas e Genéticas, SAEG versão 9.1 (Funarbe, 2007).

\section{RESULTADOS E DISCUSSÃO}

A matéria seca da parte aérea dos adubos verdes do presente experimento foi similar à encontrada em vários trabalhos. Para a parte aérea de feijão de porco, valores de 2,7 t ha-1 de matéria seca em Lavras-MG (Teixeira et al., 2005), entre 4,9 e 10,2 t ha-1 (Fávero et al., 2000), 10,8 $\mathrm{tha}^{-1}$ da matéria seca (Oliveira et al., 2002) e 14,7 t ha-1 no cerrado (Carneiro et al., 2008) foram encontrados. Ao contrário disso, para $C$. juncea, valores de matéria seca encontrados são, em média, 9,5 t ha-1 (Perin et al., 2004; Castro et al., 2005). Essas espécies acumularam grande massa devido à época de corte, realizada aos 118 dias, uma vez que geralmente o corte dos adubos verdes é feito dos 60 aos 70 dias após o plantio. Outros aspectos podem estar relacionados ao aumento da massa 
Tabela 1. Valores médios de produção da massa da matéria fresca e seca (MF e MS), teor de matéria seca (TMS) e N, fixação biológica de nitrogênio (FBN), relação C:N e acúmulo de nutrientes na raiz (RA), parte aérea (PA) e planta inteira (PI) de Crotalária (CR) e Feijão de porco (FP) aos 118 dias após o plantio (average values of fresh and dry matter (MF and MS), dry matter (MS) and N content, biological nitrogen fixation (FBN), C:N ratio and nutrient accumulation in aboveground part (RA), shoot (PA) and the whole plant (PI) Sunnhemp (CR) Jack bean (FP), 118 days after planting). Viçosa, UFV, 2008.

\begin{tabular}{|c|c|c|c|c|c|c|}
\hline \multirow{2}{*}{ Tratamentos } & MF & MS & TMS & $\mathbf{N}$ & FBN & $C: N$ \\
\hline & \multicolumn{2}{|c|}{ (t/ha) } & \multicolumn{4}{|c|}{$(\%)$} \\
\hline CRRA & $(105,34)$ & $(28,13)$ & $(26,70)$ & $(0,96)$ & 46,10 & $(42,70)$ \\
\hline CRPA & 114,51 & 30,58 & 26,70 & 1,74 & 46,10 & 23,56 \\
\hline CRPI & 219,85 & 58,71 & 26,70 & 1,37 & 46,10 & 29,92 \\
\hline FPRA & $(24,75)$ & $(4,98)$ & $(20,12)$ & $(2,48)$ & 55,49 & $(16,53)$ \\
\hline FPPA & 99,03 & 19,94 & 20,13 & 2,51 & 55,49 & 16,33 \\
\hline FPPI & 123,78 & 24,92 & 20,13 & 2,50 & 55,49 & 16,40 \\
\hline \multirow{2}{*}{ Tratamentos } & $\mathbf{N}$ & $\mathbf{P}$ & $\mathbf{K}$ & $\mathrm{Ca}$ & Mg & $S$ \\
\hline & \multicolumn{6}{|c|}{ (kg/ha) } \\
\hline CRRA & $(283,56)$ & $(38,20)$ & $(111,16)$ & $(76,40)$ & $(35,26)$ & $(70,52)$ \\
\hline CRPA & 530,82 & 71,87 & 345,60 & 275,25 & 58,11 & 43,58 \\
\hline CRPI & 814,38 & 110,07 & 457,26 & 351,65 & 93,37 & 114,10 \\
\hline FPRA & $(123,63)$ & $(11,71)$ & $(42,87)$ & $(29,66)$ & $(13,46)$ & $(10,46)$ \\
\hline FPPA & 504,32 & 44,86 & 293,14 & 277,69 & 31,90 & 28,91 \\
\hline FPPI & 627,95 & 56,57 & 336,01 & 307,35 & 45,36 & 39,37 \\
\hline
\end{tabular}

( ) Valores entre parêntesis foram obtidos pela relação raiz:parte aérea (0,92 para crotalária e 0,25 para feijão de porco), dos teores de nutrientes e relação C:N obtidos em experimento em vasos (Vargas, 2009) (values in brackets were obtained by root:shoot ratio (0.92 for sunnhemp and 0.25 for jack bean), nutrient content and C:N ratio obtained from pot experiment).

dos adubos verdes, como solos de alta fertilidade, naturalmente destinados ao cultivo de hortas, em conjunto com a época adequada ao seu cultivo.

Quanto à planta inteira, a produção estimada de matéria seca das plantas inteiras (raiz + parte aérea), foi estimada em 58,71 e 24,92 t ha ${ }^{-1}$ para crotalária e feijão de porco, respectivamente (Tabela 1). Isso pode ser explicado pelo alto potencial de adaptação da crotalária às condições edafoclimáticas da Zona da Mata Mineira (Perin et al., 2006). O baixo teor de $\mathrm{N}(0,96 \%)$ na crotalária resultou em maior relação $\mathrm{C} / \mathrm{N}(42,70)$ nas raízes de crotalária em relação ao feijão de porco.

A crotalária apresentou acúmulo de $\mathrm{N}$ na planta inteira mais elevado do que o feijão de porco (Tabela 1). Esses dados são semelhantes aos obtidos por Fontanétti et al. (2006), que verificaram os maiores acúmulos de $\mathrm{N}$ na parte aérea de crotalária $\left(374,85 \mathrm{~kg} \mathrm{ha}^{-1}\right)$ em relação ao feijão de porco $\left(246,95 \mathrm{~kg} \mathrm{ha}^{-1}\right)$.

Os acúmulos mais elevados de $\mathrm{P}, \mathrm{Ca}$, $\mathrm{Mg}$ e $\mathrm{S}$ foram observados na crotalária devido à grande produção de massa (Tabela 1). Ao contrário disso, para o enxo- fre, os dados de Fontanétti et al. (2006) relatam maiores acúmulos em plantas de feijão de porco. Com relação ao $\mathrm{K}$, ambas espécies apresentaram acúmulo de quantidades elevadas do nutriente.

Os dados mostraram que a adubação verde com leguminosas é um efetivo suprimento de $\mathrm{N}$ para as hortaliças. Destaca-se que, a partir do processo de FBN (Tabela 1), as plantas inteiras de crotalária e de feijão de porco forneceram ao solo $375,43(46,10 \%)$ e 348,45 $(55,49 \%) \mathrm{kg} \mathrm{ha}^{-1} \mathrm{de} \mathrm{N}$ proveniente do ar, respectivamente.

Para o primeiro cultivo de repolho com $50 \%$ da dose recomendada de adubação mineral nitrogenada (AM $\mathrm{N}$ ), acrescidos da raiz ou parte aérea de ambas as leguminosas, resultou em produção de cabeça similar à obtida com o fornecimento de $100 \% \mathrm{AMN}(\mathrm{p} \geq 0,05)$. O cultivo com $50 \%$ AM N acrescido da planta inteira de crotalária, aumentou a produção em relação às plantas cultivadas com 100\% AM N (Tabela 2).

$\mathrm{O}$ rendimento de cabeça variou de 1,87 a $2,33 \mathrm{~kg} /$ planta, o qual corresponde à produtividade de $47 \mathrm{a} 58 \mathrm{t} \mathrm{ha}^{-1}$. Esses resultados foram superiores aos obtidos por Fontanétti et al. (2006), que trabalharam com pré-cultivos de $C$. juncea acrescido de composto orgânico (20 t ha $^{-1}$ matéria fresca) ou vegetação espontânea com adubação mineral, relatando médias de $1,37 \mathrm{~kg}$ e 1,96 kg de matéria fresca comercial de cabeças de repolho, respectivamente. Resultados semelhantes foram encontrados por Oliveira et al. (2005), que em áreas de pré-cultivos de crotalária, obtiveram médias de $1,26 \mathrm{~kg}$ de matéria fresca comercial de cabeças de repolho. A superioridade na produção de repolho, e na produção e no acúmulo de nutrientes das leguminosas no presente trabalho em relação aos trabalhos citados se deve à grande quantidade de nutrientes reciclados e translocados de camadas mais profundas do solo pelas leguminosas e à elevada fertilidade do solo.

Os teores de $\mathrm{N}$ na parte aérea das plantas de repolho variaram de 2,70 a $3,64 \%$. O maior teor de $\mathrm{N}$ foi relacionado ao tratamento com a aplicação da planta inteira de crotalária, ocorrendo diferença deste tratamento com a testemunha AM100, conforme descrito por Vargas (2009).

A produção de massa da matéria 
Tabela 2. Valores médios de produção da massa da matéria fresca e seca de cabeças de repolho (MFCAB e MSCAB) e total da parte aérea (MFTOTAL e MSTOTAL), aos 92 dias após o transplantio e comparação à testemunha AM100 e AM50 no primeiro cultivo (average values of fresh and dry head matter of cabbage head (MFCAB and MSCAB) and total aboveground part (MFTOTAL and MSTOTAL) at 92 days after planting (DAP) and compared to the control AM100 and AM50 on the first crop). Viçosa, UFV, 2008.

\begin{tabular}{|c|c|c|c|c|}
\hline \multirow{2}{*}{ Tratamentos } & MFCAB & MSCAB & MFTOTAL & MSTOTAL \\
\hline & \multicolumn{4}{|c|}{ (g/planta) } \\
\hline AM100 & 2010,53 & 121,32 & 3443,09 & 260,61 \\
\hline AM50 & 2080,25 & 121,88 & 3603,86 & 273,72 \\
\hline CRRA AM50 & 1969,72 & 131,78 & 3343,23 & 287,88 \\
\hline CRPA AM50 & 1957,75 & 119,75 & 3571,33 & 271,25 \\
\hline CRPI AM50 & $2333,91 \mathrm{a} \mathrm{b}$ & $139,75 \mathrm{a} \mathrm{b}$ & $4190,81 \mathrm{a} \mathrm{b}$ & $316,80 \mathrm{a} \mathrm{b}$ \\
\hline FPRA AM50 & $1867,64 \mathrm{~b}$ & 122,41 & 3220,00 & 262,79 \\
\hline FPPA AM50 & 2021,61 & 118,33 & 3534,34 & 268,23 \\
\hline FPPI AM50 & 2076,46 & 124,27 & 3759,58 & $296,67 \mathrm{a}$ \\
\hline $\mathrm{CV}(\%)$ & 5,28 & 5,08 & 6,36 & 6,64 \\
\hline DMS & 190,79 & 11,23 & 403,12 & 32,87 \\
\hline
\end{tabular}

Nas colunas as médias seguidas de (a) diferem da testemunha $100 \%$ e (b) da testemunha $50 \%$ de adubação de $\mathrm{N}$ pelo teste de Dunnett $(\mathrm{p} \leq 0,05)$ (in columns, means followed by (a) differ from the control $100 \%$ and (b) the control $50 \%$ of $N$ fertilizer by the Dunnett test $(\mathrm{p} \leq 0.05))$.

fresca ou seca da parte aérea apresentou o mesmo comportamento da produção de cabeça (Tabela 2). O valor médio de diâmetro horizontal da cabeça foi de $19,86 \mathrm{~cm}$, não havendo diferença significativa comparativamente à testemunha $(\mathrm{p} \geq 0,05)$.

Plantas de repolho cultivadas em solo com raiz de crotalária, planta inteira de feijão de porco e parte aérea de ambas as espécies de leguminosas resultaram em produções similares àquelas cultivadas com 50\% AM N (Tabela 2). Mesmo o acúmulo de mais de $500 \mathrm{~kg} \mathrm{ha}^{-1}$ de $\mathrm{N}$ (Tabela 1) na parte aérea dos adubos verdes não resultou em produções superiores à adubação mineral reduzida em 50\%. Desse modo, a aplicação do $\mathrm{N}$-leguminosa pode ter promovido, indiretamente, maior aproveitamento do $\mathrm{N}$ do solo e do fertilizante pelo repolho devido à pronta disponibilidade do $\mathrm{N}$-fertilizante e/ou do $\mathrm{N}$ orgânico do solo (Muraoka et al., 2002). Apesar do grande aporte de $\mathrm{N}$ pelas leguminosas, a disponibilidade de $\mathrm{N}$ em formas absorvíveis pela planta de repolho pode ter ocorrido fora da época de maior exigência do nutriente. Nesse sentido Matos et al. (2008) relatam que após a incorporação de quatro espécies de leguminosas (A. pintoi, C. mucunoides, S. aterrimum e $S$. guianensis) plantadas no verão num Latossolo Vermelho- fresca de cabeça (Tabela 3). Em relação às demais variáveis houve diferenças somente devido à parte da planta. A aplicação da planta inteira de crotalária proporcionou maiores produções de cabeças de repolho quando comparado com a aplicação da parte aérea ou da raiz (Tabela 3). Plantas de repolho cultivadas em solo com resíduos de planta inteira de feijão de porco resultaram em produções de matéria fresca similares àquelas de plantas cultivadas com a parte aérea, mas superiores à daquelas cultivadas com raiz (Tabela 3 ).

Plantas de repolho cultivadas sob o efeito de parte aérea das leguminosas apresentaram produções de matéria seca de cabeças inferiores, comparativamente às cultivadas sob os efeitos de planta inteira e raiz (Tabela 3). Por outro lado, a matéria fresca e seca total das plantas de repolho foi superior quando as plantas foram cultivadas com a aplicação de planta inteira de leguminosas. Plantas de repolho cultivadas em solo com resíduos de planta inteira resultaram em diâmetros de cabeça similares àqueles de plantas cultivadas com a parte aérea, mas superiores às daquelas cultivadas com raiz. Estes resultados indicam que, apesar da remoção de nutrientes dos solos, pela retirada da parte aérea, nos tratamentos com raiz de leguminosas, as plantas cultivadas nestes ambientes apresentaram desempenho similar ao de plantas cultivadas sobre resíduos da parte aérea (Tabela 3 ). Tal fato pode ser explicado pelos nutrientes das raízes já se encontrarem no solo e, os nutrientes provenientes da parte aérea, principalmente o N, possam ter-se volatilizado.

No segundo cultivo, plantas de repolho cultivadas sob a influência do efeito residual de parte aérea de crotalária ou da planta inteira de ambas as leguminosas resultaram em produtividade superior àquela obtida com o efeito residual de $100 \%$ AM N (Tabela 4), enquanto que os demais tratamentos não diferiram da testemunha. $\mathrm{O}$ rendimento variou de 1,36 a $1,99 \mathrm{~kg} /$ planta, os quais correspondem à produtividade entre 34 a $50 \mathrm{t} \mathrm{ha}^{-1}$ (Tabela 4). Praticamente inexistem trabalhos que avaliem o efeito residual de leguminosas por dois ciclos produtivos consecutivos e que auxiliem na discussão dos resultados. 
Tabela 3. Valores médios da massa da matéria fresca e seca de cabeças de repolho (MFCAB e MSCAB), total da parte aérea (MFTOTAL e MSTOTAL) das plantas e do diâmetro horizontal (DIAM) das cabeças em função das partes e do efeito residual da raiz (RA), parte aérea (PA) e planta inteira (PI), e das leguminosas, C. juncea e C. ensiformis (average values of fresh and dry head matter of cabbage head (MFCAB and MSCAB), total aboveground part (MFTOTAL and MSTOTAL) of plants and horizontal diameter of the head (DIAM) according to the parts and to the residual effect of root (RA), aboveground part (PA) and the whole plant (PI), and legumes, C. juncea and C. ensiformis). Viçosa, UFV, 2008.

\begin{tabular}{|c|c|c|c|c|c|c|}
\hline \multirow{4}{*}{ Partes } & \multicolumn{5}{|c|}{$1^{\circ}$ Cultivo } & \multirow{4}{*}{ DIAM (cm) } \\
\hline & \multicolumn{2}{|c|}{ MFCAB } & \multirow[t]{2}{*}{ MSCAB } & \multirow[t]{2}{*}{ MFTOTAL } & \multirow[t]{2}{*}{ MSTOTAL } & \\
\hline & C. juncea & C. ensiformis & & & & \\
\hline & \multicolumn{5}{|c|}{ (g/planta) } & \\
\hline PI & $2333,9 \mathrm{aA}$ & $2076,4 \mathrm{aB}$ & $132,0 \mathrm{a}$ & $3975,1 \mathrm{a}$ & 306,7 a & $20,4 \mathrm{a}$ \\
\hline PA & $1957,7 \mathrm{bA}$ & $2021,6 \mathrm{abA}$ & $119,0 \mathrm{~b}$ & $3552,8 \mathrm{~b}$ & $269,7 \mathrm{~b}$ & $19,8 \mathrm{ab}$ \\
\hline RA & $1969,7 \mathrm{bA}$ & $1867,6 \mathrm{bA}$ & $127,1 \mathrm{a}$ & $3281,6 \mathrm{~b}$ & $275,3 \mathrm{~b}$ & $19,3 \mathrm{~b}$ \\
\hline $\mathrm{CV}(\%)$ & 5,28 & 5,28 & 5,08 & 6,36 & 6,64 & 3,60 \\
\hline \multirow{3}{*}{ Partes } & \multicolumn{5}{|c|}{$2^{\circ}$ Cultivo (Efeito residual) } & \\
\hline & MFC & & MSCAB & MFTOTAL & MSTOTAL & \multirow{2}{*}{ DIAM (cm) } \\
\hline & \multicolumn{4}{|c|}{ (g/planta) } & & \\
\hline PI & \multicolumn{2}{|c|}{$1941,94 \mathrm{a}$} & 116,25 a & $2829,27 \mathrm{a}$ & 204,06 a & $18,16 \mathrm{a}$ \\
\hline PA & \multicolumn{2}{|c|}{$1712,22 \mathrm{~b}$} & $102,52 \mathrm{~b}$ & $2524,99 \mathrm{~b}$ & $180,90 \mathrm{~b}$ & $17,40 \mathrm{a}$ \\
\hline RA & \multicolumn{2}{|c|}{$1457,98 \mathrm{c}$} & $96,53 \mathrm{~b}$ & $2202,73 \mathrm{c}$ & $177,34 \mathrm{~b}$ & $16,28 \mathrm{~b}$ \\
\hline $\mathrm{CV}(\%)$ & \multicolumn{2}{|c|}{10,87} & 10,82 & 9,06 & 8,57 & 5,17 \\
\hline
\end{tabular}

Médias seguidas por letras distintas, minúsculas na coluna e maiúsculas na linha, diferem entre si pelo teste de Tukey (p $\geq 0,05$ ) (means followed by different letters, 'lowercase in columns and uppercase in lines, differ by Tukey test $(\mathrm{p} \geq 0.05)$ ).

Tabela 4. Valores médios de produção da massa da matéria fresca e seca de cabeças de repolho (MFCAB e MSCAB), da parte aérea (MFTOTAL e MSTOTAL) e diâmetro horizontal (DIAM) da cabeça aos 118 dias após o transplantio e comparação à testemunha AM100 e AM50 no segundo cultivo (average values of fresh and dry head matter of cabbage head (MFCAB and MSCAB), total aboveground part (MFTOTAL and MSTOTAL) and horizontal diameter (DIAM) of the head, 92 days after planting (DAP) and compared to the control AM100 and AM50 on the second crop). Viçosa, UFV, 2008.

\begin{tabular}{|c|c|c|c|c|c|}
\hline \multirow{2}{*}{ Tratamentos } & MFCAB & MSCAB & MFTOTAL & MSTOTAL & \multirow{2}{*}{ DIAM (cm) } \\
\hline & \multicolumn{4}{|c|}{ (g/planta) } & \\
\hline AM100 & 1367,79 & 82,54 & 2068,53 & 150,66 & 15,7 \\
\hline AM50 & 1547,92 & 90,69 & 2329,25 & 168,57 & 16,5 \\
\hline CRRA AM50 & 1421,02 & 95,07 & 2140,80 & 176,81 & 16,1 \\
\hline CRPA AM50 & $1747,12 \mathrm{a}$ & $106,87 \mathrm{a}$ & $2575,07 \mathrm{a}$ & $184,56 \mathrm{a}$ & $17,5 \mathrm{a}$ \\
\hline CRPI AM50 & $1892,50 \mathrm{ab}$ & $113,32 \mathrm{ab}$ & $2779,33 \mathrm{a} b$ & $198,07 \mathrm{a} \mathrm{b}$ & $18,2 \mathrm{ab}$ \\
\hline FPRA AM50 & 1494,94 & 97,99 & 2264,66 & 177,88 & 16,3 \\
\hline FPPA AM50 & 1677,31 & 98,18 & $2474,90 \mathrm{a}$ & 177,24 & 17,2 \\
\hline FPPI AM50 & $1991,39 \mathrm{a} \mathrm{b}$ & $119,18 \mathrm{a} b$ & $2879,20 \mathrm{a} \mathrm{b}$ & $210,05 \mathrm{a} \mathrm{b}$ & $18,0 \mathrm{a}$ \\
\hline $\mathrm{CV}(\%)$ & 10,87 & 10,82 & 9,06 & 8,57 & 5,17 \\
\hline DMS & 315,86 & 19,23 & 390,91 & 27,36 & 1,55 \\
\hline
\end{tabular}

Nas colunas, médias seguidas de (a) diferem da testemunha $100 \%$ e (b) da testemunha $50 \%$ de adubação de N pelo teste de Dunnett (p $\geq 0,05$ ) (in columns, means followed by (a) differ from the control $100 \%$ and (b) the control $50 \%$ of $\mathrm{N}$ fertilizer by the Dunnett test ( $\mathrm{p} \geq 0.05$ )).

A produção da massa da matéria fresca total das plantas de repolho seguiu o mesmo comportamento da produção de cabeças (Tabela 4).

A massa da matéria seca total e o diâmetro horizontal da cabeça de repolho foram influenciados positivamente pelo efeito residual de parte aérea de crotalária e da planta inteira de ambas as espécies quando comparadas às plantas cultivadas com $100 \%$ de AM N (Tabela 4). Em trabalhos realizados com pré-cultivos de crotalária para a produção do repolho, Fontanétti et al. (2006) e Oliveira et al. (2005) obtiveram médias de diâmetro horizontal entre 15 a 19 cm, semelhantes às do presente experimento onde apenas há o efeito residual das partes de leguminosas e do N-fertilizante do cultivo agrícola anterior.

Plantas cultivadas sobre o efeito residual da aplicação da planta inteira de quaisquer das leguminosas resultaram em produtividade superior àquela 
obtida em cultivo sobre o efeito residual de 50\% da AM N (Tabela 4). O relativo aproveitamento a longo prazo da adubação verde pelas plantas de repolho sugere que o efeito residual deva ser maior do que o do fertilizante inorgânico aplicado. A incorporação de massa de leguminosas ao solo, sob condições de laboratório, resultou em mineralização líquida de $\mathrm{N}$ nas duas primeiras semanas, seguidas no entanto, de 5 semanas de imobilização do nutriente e posterior mineralização após a sétima semana (Matos et al., 2008). Assim, o N proveniente das leguminosas segue dinâmica mais complexa no solo e pode estar disponível em períodos mais longos de tempo (Muraoka et al., 2002), possibilitando importante efeito sobre outros ciclos produtivos que não aquele imediatamente subsequente às leguminosas.

Quando se compara a testemunha de $50 \%$ da dose recomendada de $\mathrm{N}$ com os demais tratamentos, verifica-se apenas que a massa da matéria fresca e seca total das plantas de repolho foi influenciada pelo efeito da aplicação de planta inteira de ambas as espécies de leguminosas e que o diâmetro horizontal foi influenciado apenas pela aplicação da planta inteira de crotalária (Tabela 4).

$\mathrm{Na}$ comparação entre as leguminosas no segundo cultivo, plantas de repolho cultivadas sobre o efeito residual da planta inteira de quaisquer das leguminosas resultaram em produções superiores àquelas cultivadas em raiz ou parte aérea (Tabela 3 ). Dentro dessa perspectiva, o efeito da raiz fica evidenciado quando combinado com o efeito de parte aérea, ou seja, no segundo ciclo produtivo a combinação $\mathrm{RA}+\mathrm{PA}$ resultou em maiores produções de repolho quando comparados com os demais tratamentos. Provavelmente, os altos valores de $\mathrm{C}: \mathrm{N}$, principalmente nas raízes de crotalária (Tabela 1), possam ter contribuído para uma mineralização mais tardia. Em adição, o melhor desempenho do combinado RA+PA possa ser explicado pela menor volatilização de $\mathrm{N}$ das raízes, por estar incorporada ao solo.

Em estudos de perdas de $\mathrm{N}$ por volatilização com a aplicação de doses de adubos verdes, Janzen \& Mcginn (1991) observaram que as perdas mais elevadas foram observadas com a aplicação da maior dose de mucuna, provavelmente pela formação de amônia diretamente na massa vegetal em decomposição, devido a sua alcalinização resultando em volatilização sem passar pelo solo. Por outro lado, em trabalho realizado com milho e brócolis cultivados em sequência à adubação verde, Perin et al. (2004) relatam que o pré-cultivo com crotalária resultou em pequeno aproveitamento do N-FBN pelo brócolis e o efeito residual não resultou em maiores diâmetro das inflorescências ou produções.

As quantidades de nutrientes na massa das leguminosas foram superiores nos tratamentos com a aplicação da planta inteira de ambas as espécies (Tabela 3). A maior oferta de outros nutrientes, que não o $\mathrm{N}$, que certamente foram mineralizados, provavelmente reflete nas maiores produções do repolho no segundo ciclo produtivo quando comparados com os tratamentos que levaram apenas a parte aérea ou raiz de leguminosas.

De acordo com os resultados alcançados, o cultivo sobre o sistema radicular de feijão de porco reduz a produtividade de repolho. Com o cultivo sobre a massa da parte aérea ou da planta inteira de crotalária ou feijão de porco reduz a necessidade de adubação com $\mathrm{N}$-mineral em 50\%. Áreas em que foram deixadas a parte aérea ou a planta inteira de crotalária ou feijão de porco resultaram em maiores efeitos residuais sobre o segundo cultivo do que a adubação com $\mathrm{N}$-mineral. O cultivo sucessivo sobre as raízes destas leguminosas não resulta em efeito residual diferente do proveniente da adubação mineral com 50 ou 100\% da recomendação da adubação $\mathrm{N}$.

\section{AGRADECIMENTOS}

À Fundação de Amparo à Pesquisa do estado de Minas Gerais, pela concessão de bolsa ao primeiro autor e ao CNPq pelo auxílio financeiro à pesquisa.

\section{REFERÊNCIAS}

BODDEY RM; ALVES BJR; URQUIAGA S. 1994. Quantificação da fixação biológica de nitrogênio associada a plantas utilizando o isótopo 15N. In: HUNGRIA M; ARAÚJO RS (eds). Manual de métodos empregados em estudos de microbiologia agrícola. Brasília:
Embrapa-CNPAF. p. 471-494.

CARNEIRO MAC; CORDEIRO MAS; ASSIS PCR; MORAES ES; PEREIRAHS; PAULINO HB; SOUZA ED. 2008. Produção de fitomassa de diferentes espécies de cobertura e suas alterações na atividade microbiana de solo de cerrado. Bragantia 67: 455-462.

CASTRO CM; ALMEIDA DL; RIBEIRO RLD; CARVALHO JF. 2005. Plantio direto, adubação verde e suplementação com esterco de aves na produção orgânica de berinjela. Pesquisa Agropecuária Brasileira 40: 495502.

DINIZ ER; SANTOS RHS; URQUIAGA SS; PETERNELLI LA; BARRELLA TP; FREITAS GB. 2007. Green manure incorporation timing for organically grown broccoli. Pesquisa Agropecuária Brasileira 42: 199-206.

EMBRAPA. 1999. Centro Nacional de Pesquisa de Solos. Sistema Brasileiro de Classificação de Solos. Rio de Janeiro. 412p.

FÁVERO C; JUCKSCH I; COSTA LM; ALVARENGA RC; NEVES JCL. 2000. Crescimento e acúmulo de nutrientes por plantas espontâneas e por leguminosas utilizadas para adubação verde. Revista Brasileira de Ciência do Solo 24: 171-177.

FONTANÉTTI A; CARVALHO GJ; GOMES LAA; ALMEIDA K; MORAES SRG; TEIXEIRA CM. 2006. Adubação verde na produção orgânica de alface americana e repolho. Horticultura Brasileira 24: 146-150.

FUNDAÇÃO ARTHUR BERNARDES. 2007. SAEG Sistema para análises estatísticas. Versão. 9.1. Viçosa-MG.

IBGE - Instituto Brasileiro de Geografia e Estatística. 2011, 21 de fevereiro. Sistema IBGE de Recuperação Automática (SIDRA). Disponível em http://www.sidra.ibge.gov.br/.

JANZEN HH; MCGINN SM. 1991. Volatile loss of nitrogen during decomposition of legume green manure. Soil Biology and Biochemistry 23: 291-297.

JENSEN ES. 1996. Rhizodeposition of $\mathrm{N}$ by pea and barley and its effect on soil N dynamics. Soil Biology \& Biochemistry 28: 65-71.

KHAN DF; PEOPLES MB; HERRIDGE DF. 2002. Quantifying below-ground nitrogen of legumes. 1. Optimising procedures for $15 \mathrm{~N}$ shoot-labelling. Plant and Soil 245: 327-334.

MATOS ES; MENDONÇA ES; LIMA PC; COELHO MS; MATEUS RF; CARDOSO IM. 2008. Green manure in coffee systems in the region of Zona da Mata, Minas Gerais: characteristics and kinetics of carbon and nitrogen mineralization. Revista Brasileira de Ciência do Solo 32: 2027-2035.

MAYER J; BUEGGER F; JENSEN ES; SCHLOTER M; HEB J. 2003. Estimating N rhizodeposition of grain legumes using a $15 \mathrm{~N}$ in situ stem labelling method. Soil Biology \& Biochemistry 35: 21-28.

MURAOKA T; AMBROSANO EJ; ZAPATA F; BORTOLETTO N; MARTINS ALM; TRIVELIN PCO; BOARETTO AE; SCIVITTARO WB. 2002. Eficiência de abonos verdes (crotalaria y mucuna) y urea, aplicados solos o juntamente, como fuentes de N para el cultivo de arroz. Terra 20: 17-23. 
OLIVEIRA AP; FERREIRA DS; COSTA CC; SILVA AF; ALVES EU. 2001. Uso de esterco bovino e húmus de minhoca na produção de repolho híbrido. Horticultura Brasileira 19: 70-73.

OLIVEIRA FL; RIBAS RGT; JUNQUEIRA RM; PADOVAM MP; GUERRA JGM; ALMEIDA DL; RIBEIRO RLD. 2005. Desempenho do consórcio entre repolho e rabanete com précultivo de crotalaria, sob manejo orgânico. Horticultura Brasileira 23: 184-188.

OLIVEIRA TK; CARVALHO GJC; MORAES RNS. 2002. Plantas de cobertura e seus efeitos sobre o feijoeiro em plantio direto. Pesquisa Agropecuária Brasileira 37: 1079-1087.

PEREIRA AJ. 2007. Caracterização agronômica de espécies de Crotalaria L. em diferentes condições edafoclimáticas e contribuição da adubação verde com $C$. juncea no cultivo orgânico de brássicas em sistema plantio direto. Seropédica: UFRRJ. 72p (Tese doutorado).

PERIN A; SANTOS RHS; URQUIAGA S; GUERRA JGM; CECON PR. 2004. Produção de fitomassa, acúmulo de nutrientes e fixação biológica de nitrogênio por adubos verdes em cultivo isolado e consorciado. Pesquisa Agropecuária Brasileira 39: 35-40.

PERIN A; SANTOS RHS; URQUIAGA SS; CECON PR; GUERRA JGM; FREITAS GB. 2006. Use of sunnhemp and millet as green manure for tropical maize production. Scientia Agricola 63: 453-459.

SAWATSKY N; SOPER RJ. 1991. A quantitative measurement of the nitrogen loss from the root system of field peas (Pisum arvense L.) grown in the soil. Soil Biology \& Biochemistry 23: $255-259$.

SOUZA JL; REZENDE P. 2006. Manual de Horticultura Orgânica. 2 ed. Viçosa: Aprenda
Fácil. 843p.

TEDESCO MJ; GIANELLO C; BISSANI CA; BOHNEN H; VOLKWEISS SJ. 1995. Análises de solo, plantas e outros materiais. 2. ed. Porto Alegre: UFRGS. 174p. (Boletim técnico, 5).

TEIXEIRA CM; CARVALHO GJ; NETO AEF; ANDRADE MJB; MARQUES ELS. 2005. Produção de biomassa e teor de macronutrientes do milheto, feijão-de-porco e guandu-anão em cultivo solteiro e consorciado. Ciência e Agrotecnologia 29: 93-99.

VARGAS TO. 2009. Contribuição da raiz e da parte aérea de duas leguminosas de adubação verde na produção de repolho. Viçosa: UFV. 85p. (Tese mestrado).

VIDIGAL SM; PEREIRA PRG; PEDROSA MW. 2007. Repolho. In: TRAZILBO JÚNIOR JP; MADELAINE V. (eds). 101 Culturas: manual de tecnologias agrícolas. Belo Horizonte: EPAMIG. p. 665-674. 